\title{
Standardized Reporting System Use During Handoffs Reduces Patient Length of Stay in the Emergency Department
}

\author{
Robert T. Dahlquist ${ }^{\mathrm{a}}$, Karina Reyner ${ }^{\mathrm{a}}$, Richard D. Robinson ${ }^{\mathrm{b}}$, Ali Farzad ${ }^{\mathrm{a}}$, \\ Jessica Laureano-Phillips ${ }^{\mathrm{c}}$, John S. Garrett ${ }^{\mathrm{a}}$, Joseph M. Young ${ }^{\mathrm{a}}$, \\ Nestor R. Zenarosa ${ }^{\mathrm{b}}$, Hao Wang ${ }^{\mathrm{b}, \mathrm{d}}$
}

\begin{abstract}
Background: Emergency department (ED) shift handoffs are potential sources of delay in care. We aimed to determine the impact that using standardized reporting tool and process may have on throughput metrics for patients undergoing a transition of care at shift change.

Methods: We performed a prospective, pre- and post-intervention quality improvement study from September 1 to November 30, 2015. A handoff procedure intervention, including a mandatory workshop and personnel training on a standard reporting system template, was implemented. The primary endpoint was patient length of stay (LOS). A comparative analysis of differences between patient LOS and various handoff communication methods were assessed pre- and post-intervention. Communication methods were entered a multivariable logistic regression model independently as risk factors for patient LOS.

Results: The final analysis included 1,006 patients, with 327 comprising the pre-intervention and 679 comprising the post-intervention populations. Bedside rounding occurred $45 \%$ of the time without a standard reporting during pre-intervention and increased to $85 \%$ of the time with the use of a standard reporting system in the post-intervention period $(\mathrm{P}<0.001)$. Provider time (provider-initiated care to patient care completed) in the pre-intervention period averaged 297 min, but decreased to $265 \mathrm{~min}$ in the post-intervention period $(\mathrm{P}<$ 0.001). After adjusting for other communication methods, the use of a standard reporting system during handoff was associated with short-
\end{abstract}

Manuscript submitted January 31, 2018, accepted February 19, 2018

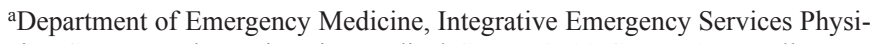
cian Group, Baylor University Medical Center, 3500 Gaston Ave, Dallas, TX 75246, USA

bDepartment of Emergency Medicine, Integrative Emergency Services Physician Group, John Peter Smith Health Network, 1500 S Main St, Fort Worth, TX 76104, USA

'Department of Emergency Medicine, Office of Clinical Research, John Peter Smith Health Network, 1500 S Main St, Fort Worth, TX 76104, USA

${ }^{\mathrm{d} C}$ Corresponding Author: Hao Wang, Department of Emergency Medicine, Integrative Emergency Services Physician Group, John Peter Smith Health Network, 1500 S Main St, Fort Worth, TX 76104, USA.

Email: hwang01@jpshealth.org

doi: https://doi.org/10.14740/jocmr3375w ened ED LOS $(\mathrm{OR}=0.60,95 \%$ CI $0.40-0.90, \mathrm{P}<0.05)$.

Conclusions: Standard reporting system use during emergency physician handoffs at shift change improves ED throughput efficiency and is associated with shorter ED LOS.

Keywords: Emergency department; Handoff; Standard reporting system

\section{Introduction}

Patient handoffs in the emergency department (ED) are a complex yet common practice for emergency physicians (EP) involving the transition of patient care from one provider to another. These transitions are often fraught with frequent interruptions, breakdowns in communication, and loss of critical data transfer, thereby introducing opportunities for medical errors, delays in care and disposition, and medicolegal liabilities $[1,2]$. Poor communication between caretakers is a major contributor to many medical errors and could potentially lead to delays in care and sentinel events $[3,4]$. Bedside handoff is known to be one of the most common and effective communications servicing transitions between providers $[5,6]$. Additional studies have shown that patient care transition is more consistent and systemic with the implementation of standard reporting systems $[7,8]$. Aside from using a systemic report, identifying potentially high-risk patients during handoff procedures seems to play a critical role in reducing medical errors and improving outcomes [9-11]. Although prior studies have evaluated the use of standardized reporting systems between different specialties, there is scant literature evaluating standardized bedside handoff procedures in an ED setting [12-15].

Bedside handoff, along with other traditional communication handoffs, are reported in the literature and include verbal discussion between physicians, written documentation via paper/charts, and/or electronic medical record (EMR) documentation $[16,17]$. Despite incremental improvements to patient quality of care, regardless of communication method employed, few studies directly link the various handoff methods to patient outcomes $[16,17]$. 
We implemented a standard reporting system template for handoff procedures during shift changes and provided a mandatory training workshop for all EPs emphasizing the need for systemic reporting of potentially high-risk patients. The intervention process involved bedside handoff coupled with a systemic reporting activity. The specific traditional handoff communication method employed was at the individual EP's discretion (e.g., bedside verbal, written, or EMR with or without standard report). A quality improvement project was conducted to compare patient care outcomes before and after this intervention. We aimed to determine whether implementing mandatory bedside handoff with a standard reporting system improved throughput and patient care outcomes.

\section{Methods}

\section{Study design}

This is a single-center, prospective qualitative cohort study. The study ED is an urban tertiary referral center with level one trauma center designation and annual visits $>100,000$. Main section of the study ED is staffed by ED physicians and fast track area is staffed by advanced practice provider. The study ED is a community hospital ED with no emergency medicine residents. This study was performed at main section of ED during a 12-week period from September 1, 2015 through November 30, 2015. The same ED providers were included during the pre- and postintervention phases who all used scribes as abstractors and realtime data collectors. As a quality improvement project, a waiver of Institutional Review Board (IRB) approval was granted.

\section{Study setting and population}

We investigated the importance of patient throughput metrics (e.g. length of stay (LOS)) by conducting a patient handoff project at the study ED. The study included all intend-to-treat patients: 1) who experienced a transition of care between EPs at some point during their ED stay, 2) whose ED physicians using scribes for their documentations and collecting data for quality improvement purposes. Patients whose care was conducted and completed solely under the management of one EP (e.g., discharged, transferred, or admitted patients), whose care rendered by ED providers not using scribes, and those whose care was immediately transferred to a non-EP upon ED arrival (e.g., trauma patients) were excluded from this study. If same patients visited ED multiple times, we considered multiple encounters and analyzed separately in this study.

\section{Study protocol}

\section{Pre-intervention}

During the 6-week pre-intervention period (September 1, 2015 through October 14, 2015), neither bedside rounding nor stand- ard reporting system utilization were emphasized when patient care transitioned from one EP to another EP at shift change. During the patient handoff process, methods of transitioning care information from one EP to another were dependent on their routine practice habit. In general, three different communication methods were commonly used during patient care information transfer with or without bedside rounding. These patient care information transition methods were: 1) verbal patient care transition information between EPs (communications between outgoing and incoming physicians), 2) hard copy documented EP discussion of patient care transition information (written paper documentation from either outgoing or incoming physicians), 3 ) electronic medical record (EMR) entry of EP discussion of patient care transition information (EMR documentation from either outgoing or incoming physicians). Pragmatically, verbal communication was commonly put to use despite other transition of care communication methods used (i.e., documented). Data were collected by scribes in real-time during handoff process.

\section{Intervention}

A mandatory workshop and personnel training were completed by each EP and a standard reporting system template was introduced (Supplementary Table 1, www.jocmr.org). Workshop training emphasized the importance of systemic reporting by using the situation, background, assessment, and recommendation (SBAR) form of communication during handoff procedures. A standard reporting system template including all potentially high-risk patient characteristics was implemented to facilitate SBAR discussions during the handoff process. Mandatory bedside patient rounding using this standard reporting system template during shift changes became effective after October 14, 2015 at the study ED. Bedside rounding was defined as physicians physically at bedside with patients during handoff process and handoff communicated between physicians using SBAR format, whether information required to be recorded in paper or in EMR was not mandatory.

\section{Post-intervention}

During the subsequent 6-week post-intervention period (October 15, 2015 through November 30, 2015), All EPs were required to complete the standard reporting system template during their shift changes. Meanwhile, EPs continued to rely on individual clinical discretion when using one of the other care information transition methods. ED scribes were utilized as primary abstractors after training on an electronic data entry platform. They contemporaneously observed the handoff process and were mandatory to record data including presence or absence of bedside handoff, method(s) of information transfer, EPs involved, and time to process completion.

\section{Outcome measurements}

Our primary outcome measurement was total patient ED LOS, 
Table 1. General Information of Study Patients

\begin{tabular}{lll}
\hline & Pre-intervention $(\mathbf{n}=\mathbf{3 2 7})$ & Post-intervention $(\mathbf{n}=\mathbf{6 7 9 )}$ \\
\hline Age - years, (mean, IQR) & $51(35-62)$ & $51(37-65)$ \\
Sex - male yes, $(\mathrm{n}, \%)$ & $173(53)$ & $342(50)$ \\
ED disposition (n, \%) & & \\
$\quad$ Discharged to home & $165(50)$ & $286(42)$ \\
\multicolumn{1}{c}{ Admitted to hospital } & $155(47)$ & $367(54)$ \\
\hline Others* & $7(2)$ & $26(4)$ \\
\hline
\end{tabular}

IQR: interquartile range; n: number; ED: emergency department. *Others including transfer to other facilities, left against medical advice, or disposition to jail/police.

especially provider-to-disposition time, defined as the interval incorporating the point at which the provider initiated patient care to the point that patient care was completed (i.e., patient final disposition decision entered into the EMR by provider), hereafter referred to as provider time. Our secondary outcome measurement was peer referral quality improvement cases including: hospital and ED quality committee referral for evaluation of ED unexpected death; 72-h return to ED; procedural compliance violations; unplanned level of care transfers after admission; delays in care; and adverse events due to treatment, procedure, or medication errors (hereafter referred to as referral cases).

\section{Variables}

General patients' characteristics were analyzed and compared before and after the intervention to include age, sex, race, and ED dispositions. We measured patients' total LOS (defined as the time interval incorporating the point the patient arrived at the ED to the point that the patient physically left the ED) and provider time (previously defined). Handoff time refers to the interval time spent by EPs during handoff procedures and includes, but may not be limited to, bedside patient rounding and patient information transition and discussion. ED scribes measured handoff intervals using 5-min time ranges (e.g., 5 $10 \mathrm{~min}, 10$ - $15 \mathrm{~min}$, etc.); therefore, a median minutes value was entered for final data analysis (e.g., 7.5 min was recorded if EPs spent 5 - $10 \mathrm{~min}$ for their handoff procedures).

\section{Sample size estimation}

Sample size was estimated on the basis of reported potential quality improvement in the literature and historical metrics in the study ED. A modified Delphi survey was conducted among the physicians and ED administrators to determine the potential decrease of ED LOS after the implementation of the handoff intervention. Our survey results indicated that $10 \%$ of shortened ED LOS after the intervention could reasonably be expected. Setting the acceptable significance level $(\alpha=0.05)$ for two tailed alternative hypotheses and assigning the power of the study at $80 \%(\beta=0.2)$ with $1: 2$ allocation ratio (preversus post-intervention phases), we estimated that the sam- ple size to be 885 for this study with 295 patients enrolled in the pre-intervention and 590 patients in the post-intervention phases.

\section{Data analysis}

Student's $t$-test was used for comparison analysis for continuous variables (before and after intervention groups). Wilcoxon rank sum test was used as an alternative to Student's $t$-test for non-parametric continuous data analysis. Pearson Chi-square $\left(\chi^{2}\right)$ analysis was used to compare categorical variables. We first compared the outcome differences before and after the intervention supplemented with an outcome difference analysis on bedside rounding during handoff with or without use of the standard reporting system template. A comparison of outcome differences among the various communication methods (bedside rounding, communication with standard reporting system template, written, and EMR) was performed using a multivariable step-wise logistic regression model. The dependent outcome variable is provider time. Due to skewed outcome data (Supplementary Table 1, www.jocmr.org), provider time was dichotomized into two categories (not prolonged versus prolonged provider time). Patients whose ED provider time was approached the upper quartile $(25 \%)$ of the entire study sample were considered patients with prolonged provider time. All potential confounders including patient age, gender, race, and patient ED dispositions along with the four different communication transition methods were entered into the final regression model as independent variables. To avoid the redundant variables in the final model, Spearman correlation and a regression with variance inflation (VIF) option including all predictive variables was used. The model's goodness of fit was measured using the Hosmer-Lemeshow test. All descriptive and statistical analyses were performed using Stata 14.0 (College Station, TX). A P value less than 0.05 was considered statistically significant.

\section{Results}

During the 12-week study period, a total of 1,006 patients were enrolled including 327 patients enrolled in the pre-intervention phase and 679 patients in the post-intervention. Table 1 shows 
Table 2. Physician Compliance With Bedside Rounding Handoff Protocol Relative to Patient Outcomes

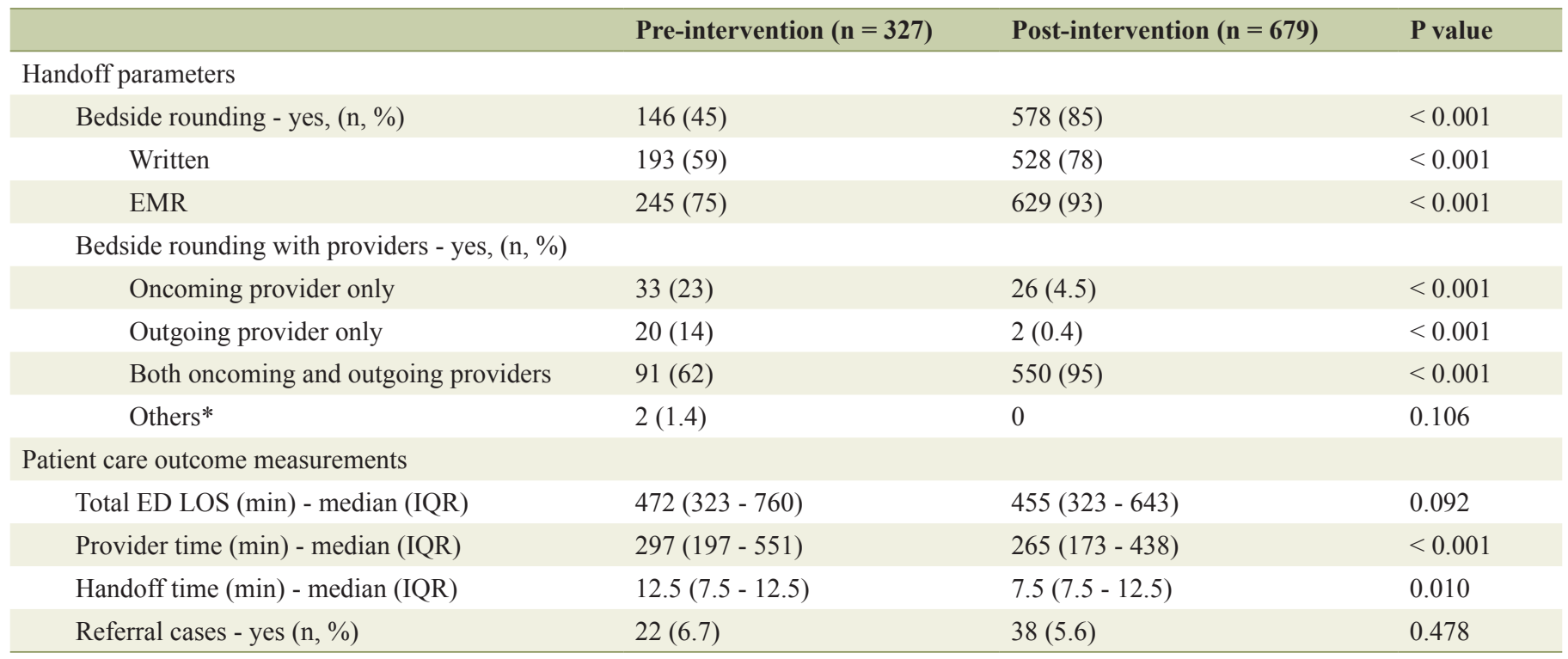

n: number; EMR: electronic medical record; ED: emergency department; LOS: length of stay; min: minutes; IQR: interquartile range. *Others including bedside rounding with nursing staff, residents, or students.

the patient population general characteristics. No statistically significant differences were reached.

Further analysis revealed that the frequency of bedside rounding increased significantly after the intervention $(45 \%$ pre- to $85 \%$ post-intervention, $\mathrm{P}<0.001$ ) regardless of communication method used during the handoff process (Table 2). The frequency of both incoming and outgoing physicians involved in the handoff process was also noted to increase significantly (37\% pre- to $84 \%$ post-intervention, $\mathrm{P}<0.001)$. Meanwhile, we found that both patient ED LOS and provider time decreased post-intervention. Although there was a trend toward improvement in referral case rate, neither this statistic nor handoff time change reached statistical significance (Table
2).

To specifically determine the role of bedside rounding with a standard reporting system template relative to improvement of patient care outcomes studied, patients were divided into two groups (patients whose bedside rounding was performed with the standard report versus those whose bedside rounding was performed without the standard report). We found that if bedside rounding with the standard report was performed during handoff, EPs tended to spend less time during the transition of care process and patients subsequently spent less time in the ED (Table 3, $\mathrm{P}<0.001$ ). Moreover, if bedside rounding with standard report was performed, fewer referral cases were reported, though the difference did not reach statistical signifi-

Table 3. Association Between Physician Bedside Rounding With and Without the Use of a Standard Reporting System Template Relative to Patient Outcomes

\begin{tabular}{|c|c|c|c|}
\hline & Pre-intervention & Post-intervention & \\
\hline & $\begin{array}{l}\text { Bedside rounding by incoming } \\
\text { and outgoing physicians without } \\
\text { standard report }(n=91)\end{array}$ & $\begin{array}{l}\text { Bedside rounding by incoming } \\
\text { and outgoing physicians with } \\
\text { standard report }(\mathrm{n}=\mathbf{5 5 0})\end{array}$ & P value \\
\hline \multicolumn{4}{|l|}{ Handoff parameters } \\
\hline \multicolumn{4}{|l|}{ Communication method - yes, (n, \%) } \\
\hline Written & $73(80)$ & $451(82)$ & 0.68 \\
\hline \multicolumn{4}{|l|}{ Patient care outcome measurements } \\
\hline Total ED LOS - min, (median, IQR) & $484(324-888)$ & $456(327-635)$ & 0.1989 \\
\hline Provider time - min, (median, IQR) & $311(226-565)$ & $263(173-435)$ & 0.005 \\
\hline Handoff time - min, (median, IQR) & $12.5(12.5-17.5)$ & $7.5(7.5-12.5)$ & $<0.001$ \\
\hline
\end{tabular}

n: number; EMR: electronic medical record; ED: emergency department; LOS: length of stay; min: minutes; IQR: interquartile range. 
Table 4. Role of Different Communication Methods Relative to Prolonged Provider Time

\begin{tabular}{llll}
\hline Communication method & Adjusted odds ratio & $\mathbf{9 5 \%}$ Confidence limit & P value \\
\hline Bedside rounding & 1.25 & $0.80-1.94$ & 0.32 \\
Standard reporting system template & 0.60 & $0.40-0.90$ & 0.01 \\
Written communication & 0.88 & $0.63-1.23$ & 0.45 \\
Electronic medical record & 0.89 & $0.58-1.38$ & 0.81 \\
\hline
\end{tabular}

ED: emergency department.

cance due to small patient sample size.

A multivariable logistic regression model was generated to determine the association of each communication method with prolonged provider time. Our findings showed that simply providing bedside rounding without using the standard reporting system template did not significantly change patient provider time. In contrast, using the standard reporting system template during handoff resulted in shorter patient ED LOS and significantly reduced provider time (Table 4).

\section{Discussion}

Appropriate handoff procedures improve patient outcomes through reduction of medical errors, recognition of unsolved problems, and improvement of patient satisfaction [18, 19]. Standard reporting systems identifying potentially high-risk patients are considered an efficient method of transitioning care but lack external comparisons and validation [20]. Currently, no study has identified best handoff practices nor has handoff standardization been mandated by regulators [21]. Mandatory training with recommendation of bedside rounding using a standard reporting system template facilitating EP handoffs was performed as part of this study. The intervention significantly improved compliance with EP bedside handoff expectations during shift change thereby decreasing provider time, an outcome which has not been reported previously. Study results demonstrate the value of performing bedside handoff in conjunction with a standard reporting system and its causal relationship in reducing provider time. Our findings add value to the literature pool by providing evidence supporting the importance of bedside rounding using a standard reporting template relative to improved patient care outcomes. Meanwhile, it further emphasizes the impact that mandatory training interventions have relative to increased compliance with ED quality improvement projects.

In this study, a quasi-experimental one group pretest and posttest study was designed with doubling the posttest sample size. This will help providing sufficient evidence against confounding. Additionally, same group of physicians entered into pre- and post-intervention phases minimized the potential variations on their routine practice. A step-wise multivariate logistic regression model further minimized the potential confounders affecting outcome measurements.

A variety of reporting systems exist and have been reported as efficient handoff procedures linked closely to improved patient care outcomes [11, 22, 23]. As SBAR is widely recommended and externally validated, similar standards were adopted at the study ED yielding similar findings [24-26]. This study did not focus on the determination and validation of this standard reporting system template's ability to identify potentially high-risk patients during the handoff procedure. We rather chose to emphasize the role of the bedside handoff itself coupled with various existing communication options commonly used for standard reporting. Our results still favor the use of a standard reporting template during handoff, regardless of additional communication methods used. These findings are consistent with previous handoff reports found among the various specialty physician literatures and further expand its value among EPs $[12,15]$. Still, our study data did not show a direct link between handoff and potential medical errors. As is known, referral cases are affected in a multifactorial manner with potential influences being physician and nursing staff, patient disease nature, ED overcrowding, etc. [27-29]. Therefore, a multi-center prospective cohort study incorporating possible medical errors risks is warranted for further investigation.

When analyzed as independent predictors, we found no significant differences between the communication methods employed in this study (i.e., bedside rounding, written, or EMR). This indicates that providers may choose to employ any of the traditional communication methods during handoffs when coupled with a standard reporting system (e.g., SBAR). A review of the current literature finds no consensus best practice recommendation when comparing the variety of handoff procedures coupled with different combinations of handoff communication tools $[4,16]$. Some studies preferred verbal communication [17] while others preferred EMR documentation [4]. One study found bedside handoff was not superior to traditional handoffs [30]. By far, comparison study on these traditional communication methods used during handoff procedures and their interaction analyses has not yet reported in the current literature. Although our results are unable to determine the superiority of one communication method to another during the handoff process, our findings establish a foundation for future transition of care communication interaction projects.

\section{Limitations}

Our study has several limitations. This is a single-center prospective study. Our study did not analyze all patient care outcomes such as patient satisfaction, 30-day readmission to hospital, or prescription errors. Additionally, the study endpoints can be affected by numerous factors, such as patient disease nature, ED crowding status, and the availability/priority of ad- 
junct services. We are therefore unable to address every potential confounder in order to minimize bias.

\section{Conclusions}

Standard reporting system use during EP handoffs in the ED significantly improves throughput without adversely affecting the transition of care time interval.

\section{Conflict of Interest}

All authors report no conflict of interest.

\section{References}

1. Cheung DS, Kelly JJ, Beach C, Berkeley RP, Bitterman RA, Broida RI, Dalsey WC, et al. Improving handoffs in the emergency department. Ann Emerg Med. 2010;55(2):171-180.

2. Kachalia A, Gandhi TK, Puopolo AL, Yoon C, Thomas EJ, Griffey R, Brennan TA, et al. Missed and delayed diagnoses in the emergency department: a study of closed malpractice claims from 4 liability insurers. Ann Emerg Med. 2007;49(2):196-205.

3. Maughan BC, Lei L, Cydulka RK. ED handoffs: observed practices and communication errors. Am J Emerg Med. 2011;29(5):502-511.

4. Gonzalo JD, Yang JJ, Stuckey HL, Fischer CM, Sanchez LD, Herzig SJ. Patient care transitions from the emergency department to the medicine ward: evaluation of a standardized electronic signout tool. Int J Qual Health Care. 2014;26(4):337-347.

5. Mardis T, Mardis M, Davis J, Justice EM, Riley Holdinsky S, Donnelly J, Ragozine-Bush H, et al. Bedside shiftto-shift handoffs: a systematic review of the literature. J Nurs Care Qual. 2016;31(1):54-60.

6. Wray CM, Arora VM, Hedeker D, Meltzer DO. Assessing the implementation of a bedside service handoff on an academic hospitalist service. Healthc (Amst). 2017.

7. Benjamin MF, Hargrave S, Nether K. Using the targeted solutions tool(R) to improve emergency department handoffs in a community hospital. Jt Comm J Qual Patient Saf. 2016;42(3):107-118.

8. Dubosh NM, Carney D, Fisher J, Tibbles CD. Implementation of an emergency department sign-out checklist improves transfer of information at shift change. J Emerg Med. 2014;47(5):580-585.

9. Hoffman RL, Saucier J, Dasani S, Collins T, Holena DN, Fitzpatrick M, Tsypenyuk B, et al. Development and implementation of a risk identification tool to facilitate critical care transitions for high-risk surgical patients. Int J Qual Health Care. 2017;29(3):412-419.

10. von Dossow V, Zwissler B. Recommendations of the German Association of Anesthesiology and Intensive Care Medicine (DGAI) on structured patient handover in the perioperative setting : The SBAR concept. Anaesthesist.
2016;65(Suppl 1):1-4.

11. Heilman JA, Flanigan M, Nelson A, Johnson T, Yarris LM. Adapting the I-PASS handoff program for emergency department inter-shift handoffs. West J Emerg Med. 2016;17(6):756-761.

12. Lee S, Jordan J, Hern HG, Kessler C, Promes S, Krzyzaniak S, Gallahue F, et al. Transition of care practices from emergency department to inpatient: survey data and development of algorithm. West J Emerg Med. 2017;18(1):86-92.

13. Moy NY, Lee SJ, Chan T, Grovey B, Boscardin WJ, Gonzales R, Pierluissi E. Development and sustainability of an inpatient-to-outpatient discharge handoff tool: a quality improvement project. Jt Comm J Qual Patient Saf. 2014;40(5):219-227.

14. Watkins LM, Patrician PA. Handoff communication from the emergency department to primary care. Adv Emerg Nurs J. 2014;36(1):44-51.

15. Apker J, Mallak LA, Applegate EB, 3rd, Gibson SC, Ham JJ, Johnson NA, Street RL, Jr. Exploring emergency physician-hospitalist handoff interactions: development of the Handoff Communication Assessment. Ann Emerg Med. 2010;55(2):161-170.

16. Arora VM, Manjarrez E, Dressler DD, Basaviah P, Halasyamani L, Kripalani S. Hospitalist handoffs: a systematic review and task force recommendations. J Hosp Med. 2009;4(7):433-440.

17. Benham-Hutchins MM, Effken JA. Multi-professional patterns and methods of communication during patient handoffs. Int J Med Inform. 2010;79(4):252-267.

18. Davis J, Roach C, Elliott C, Mardis M, Justice EM, Riesenberg LA. Feedback and Assessment Tools for Handoffs: A Systematic Review. J Grad Med Educ. 2017;9(1):18-32.

19. Downey LV, Zun L, Burke T. What constitutes a good hand offs in the emergency department: a patient's perspective. Int J Health Care Qual Assur. 2013;26(8):760767.

20. Horwitz LI, Schuster KM, Thung SF, Hersh DC, Fisher RL, Shah N, Cushing W, et al. An institution-wide handoff task force to standardise and improve physician handoffs. BMJ Qual Saf. 2012;21(10):863-871.

21. Cohen MD, Hilligoss PB. The published literature on handoffs in hospitals: deficiencies identified in an extensive review. Qual Saf Health Care. 2010;19(6):493-497.

22. Farhan M, Brown R, Woloshynowych M, Vincent C. The $\mathrm{ABC}$ of handover: a qualitative study to develop a new tool for handover in the emergency department. Emerg Med J. 2012;29(12):941-946.

23. Gopwani PR, Brown KM, Quinn MJ, Dorosz EJ, Chamberlain JM. SOUND: a structured handoff tool improves patient handoffs in a pediatric emergency department. Pediatr Emerg Care. 2015;31(2):83-87.

24. Haig KM, Sutton S, Whittington J. SBAR: a shared mental model for improving communication between clinicians. Jt Comm J Qual Patient Saf. 2006;32(3):167-175.

25. Cornell P, Gervis MT, Yates L, Vardaman JM. Improving shift report focus and consistency with the situation, background, assessment, recommendation protocol. J Nurs Adm. 2013;43(7-8):422-428. 
26. Tews MC, Liu JM, Treat R. Situation-BackgroundAssessment-Recommendation (SBAR) and Emergency Medicine Residents' Learning of Case Presentation Skills. J Grad Med Educ. 2012;4(3):370-373.

27. Hilligoss B, Cohen MD. Hospital handoffs as multifunctional situated routines: implications for researchers and administrators. Adv Health Care Manag. 2011;11:91-132.

28. Eitel DR, Rudkin SE, Malvehy MA, Killeen JP, Pines JM. Improving service quality by understanding emergency department flow: a White Paper and position statement prepared for the American Academy of Emergency Medicine. J Emerg Med. 2010;38(1):70-79.

29. Horwitz LI, Meredith T, Schuur JD, Shah NR, Kulkarni RG, Jenq GY. Dropping the baton: a qualitative analysis of failures during the transition from emergency department to inpatient care. Ann Emerg Med. 2009;53(6):701710 e 704.

30. Gosdin $\mathrm{CH}$, Vaughn L. Perceptions of physician bedside handoff with nurse and family involvement. Hosp Pediatr. 2012;2(1):34-38. 